\title{
Tip-enhanced Raman spectroscopic imaging of localized defects in carbon nanotubes
}

\author{
Carsten Georgi and Achim Hartschuh ${ }^{\text {a) }}$ \\ Department Chemie and CeNS, Ludwig-Maximilians-Universität München, 81377 München, Germany
}

(Received 15 July 2010; accepted 21 September 2010; published online 7 October 2010)

\begin{abstract}
We used tip-enhanced Raman spectroscopy to study defect induced D-band Raman scattering in metallic single-walled carbon nanotubes with a spatial resolution of $15 \mathrm{~nm}$. The spatial extent of the D-band signal in the vicinity of localized defects is visualized and found to be about $2 \mathrm{~nm}$ only. Using the strong optical fields underneath the tip, we photogenerate localized defects and derive a relation between defect density and resulting D-band intensity. (C) 2010 American Institute of Physics. [doi:10.1063/1.3499752]
\end{abstract}

Single-walled carbon nanotubes (SWNTs) are widely regarded as a versatile material for applications in nanoelectronics and nano-optics, biotechnology, and sensing. Their exceptional physical properties promise great potential, yet their actual structure is often far from perfect. Raman spectroscopy is extensively used as a nondestructive tool to study the structural properties of graphitic materials and provides information on, e.g., nanotube chirality, doping, and functionalization. ${ }^{1}$ In particular, the investigation of structural defects in carbon nanotubes is of high interest since they will strongly affect device performances. Symmetry breaking defects in graphitic materials are known to activate a dispersive phonon mode at around $1300-1350 \mathrm{~cm}^{-1}$ denoted as D-band. ${ }^{2,3}$ The intensity ratio $I_{D} / I_{G}$, with $I_{G}$ being the intensity of the G-band resulting from $\mathrm{C}=\mathrm{C}$ stretching vibrations, has been used since many years for the quantification of defect densities in $s p^{2}$ carbon structures. ${ }^{4}$

D-band scattering is connected to a large momentum phonon and cannot occur upon excitation of defect-free structures with visible light $(k \approx 0)$ due to momentum conservation. In Ref. 5 a semiclassical real space description of defect scattering in graphene has been derived, which can also be used for metallic SWNTs due to their comparable band structure for excitation energies below the first interband transition $\mathrm{M}_{11}$. Upon photoexcitation a free electron and a hole are generated with opposite momenta. Radiative recombination can occur if one charge carrier is backscattered by a D-phonon while the other is scattered back by a defect. In this real space model, the charge carrier therefore needs to reach a defect during its lifetime, which limits the length scale of the D-band scattering process $l_{D}$ near a defect. The determination of the defect density based on the D-band intensity depends critically on $l_{D}$. For graphene, a length of about $4 \mathrm{~nm}$ has been estimated based on the uncertainty principle and the electron velocity. ${ }^{5}$ A value of $2 \mathrm{~nm}$ was determined experimentally by connecting the D-band intensity with the defect density measured by scanning tunneling microscopy. ${ }^{6}$ Attempts to deduce the D-band extension near a graphene edge that acts as a one-dimensional defect, using confocal Raman microscopy were hampered by the diffraction limited spatial resolution of several $100 \mathrm{~nm} .^{7,8}$ A similar value for $l_{D}$ could be expected for metallic SWNTs. In this letter, we study D-band scattering in metallic SWNTs

${ }^{a)}$ Electronic mail: achim.hartschuh@cup.uni-muenchen.de. with subdiffraction spatial resolution of about $15 \mathrm{~nm}$ using tip-enhanced Raman scattering (TERS). ${ }^{9-11}$ We visualize the spatial extent of D-band scattering near localized defects and find that it occurs only within about $2 \mathrm{~nm}$.

Our TERS setup combines an inverted confocal microscope with a shear force mechanism to position a sharp gold tip in the center of the laser focus about $2 \mathrm{~nm}$ above the sample surface [see Fig. 1(a)]. By using a radially polarized laser beam with a wavelength of $633 \mathrm{~nm}$, excitation and radiation rates are strongly enhanced in the vicinity of the tip, which allows for the local imaging of the SWNT's Raman scattering. The laser power on the sample is $500 \mu \mathrm{W}$. The scattered light from each image pixel is detected either by a spectrograph and charge-coupled device or by an avalanche photodiode (APD) after spectral filtering. The nanotubes studied in this work were metallic arc discharge SWNTs dispersed with an ionic surfactant and sorted by density gradient ultracentrifugation (Nanointegris IsoNanotubes-M, 99\% purity). ${ }^{12}$ The aqueous solution was diluted 1:100 and spun onto a glass cover slide.

Figure 1(b) shows the near-field Raman G-band image of a $250 \mathrm{~nm}$ long SWNT, where the G-band scattering at each pixel is detected with an APD after band pass filtering. The spatial resolution of this image is around $15 \mathrm{~nm}$, determined by the apex size of the tip used here. The resolution is defined by the full width at half maximum of the Raman signal across the SWNT. Figure 1(c) shows the simultaneously acquired topography. In order to image the intensity
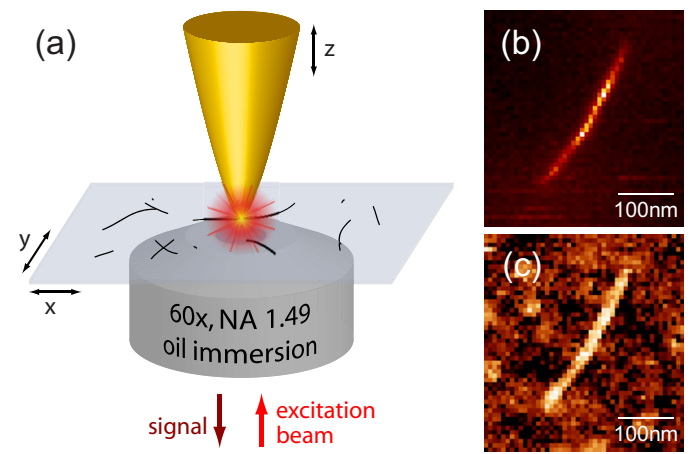

FIG. 1. (Color online) (a) Schematic of the TERS setup. (b) Near-Field Raman G-Band image of a metallic SWNT detected by an APD after band pass filtering at $1580 \pm 100 \mathrm{~cm}^{-1}$. (c) Simultaneously acquired topography (z-range $1.5 \mathrm{~nm}$ ). 

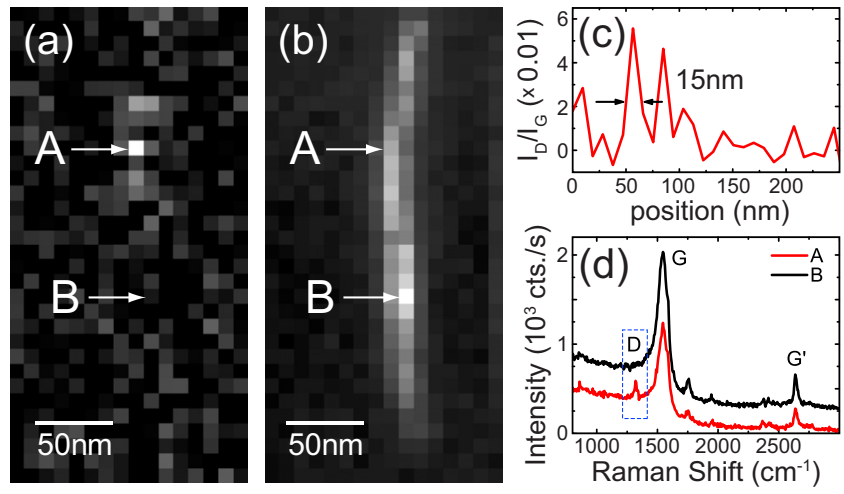

FIG. 2. (Color online) Results from a spectroscopic image of the SWNT in Fig. 1(b) showing localized D-band scattering (pixel size is $10 \mathrm{~nm}$ ). (a) D-band intensity $I_{D}$. (b) G-band intensity $I_{G}$. (c) Distribution of the $I_{D} / I_{G}$-ratio along the nanotube, showing strong local variations on length scales similar to the spatial resolution. (d) Spectra from the positions A and B marked in (a) and (b) with maximum $I_{D}$ and $I_{G}$, respectively.

of any Raman band in the spectrum, we use spectroscopic imaging where the complete spectrum is acquired at every image pixel. Subsequent data analysis then allows to create high-resolution images of selected spectral information as, e.g., Raman intensities. Figure 2 shows the results of a spectroscopic image of the SWNT in Fig. 1(b). The integrated intensity of the G-band peak $I_{G}$ in Fig. 2(b) is rather uniform along the nanotube, whereas the integrated D-band intensity $I_{D}$ [Fig. 2(a)] is strongly localized in the upper half. For quantitative discussions, the ratio of $I_{D} / I_{G}$ is commonly used as a measure for the defect concentration. The normalization by $I_{G}$ eliminates possible variations in the signal enhancement and accounts for a modified resonance condition of the Raman process due to defect induced changes in the band structure. The distribution of $I_{D} / I_{G}$ along the SWNT is plotted in Fig. 2(c), showing that the D-band scattering is localized within $15 \mathrm{~nm}$ corresponding to the spatial resolution of the experiment. Figure 2(d) presents the spectra from the indicated pixels with highest and lowest $I_{D} / I_{G}$, respectively. D-band scattering is clearly seen at position A, whereas at position $\mathrm{B}$ the spectrum indicates the absence of defects (marked with dashed box). The spatial extension of D-band scattering from a pointlike defect that is measured in the experiment arises from the convolution of the intrinsic D-band extension $l_{D}$ and the point spread function (PSF) of our experiment, represented by a Gaussian with a width corresponding to the spatial resolution. If $l_{D}$ is much smaller than the resolution, the measured width of the $I_{D} / I_{G}$-signal

along the SWNT is simply the sum of $l_{D}$ and the width of our PSF. We observe a similar value of $15 \mathrm{~nm}$ for the width of both the PSF and $I_{D} / I_{G}$. Thus, we can conclude that $l_{D}$ is very small, definitely far below $10 \mathrm{~nm}$. Here, the rather large pixel size of $10 \mathrm{~nm}$ hinders a more quantitative conclusion.

Figure 3 shows corresponding results from spectroscopic images of another SWNT on the same sample. Here, each image pixel has only $5.7 \mathrm{~nm}$ size and the local D-band intensity is stronger, giving clearer and less noisy D-band images [Figs. 3(b) and 3(c)]. In the case of this SWNT, we intentionally generated a localized defect site to study the evolution of $I_{D} / I_{G}$ and measure $l_{D}$ afterwards. Figure 3(a) shows the integrated D-band intensity from a spectroscopic image before the experiment, the respective G-band image is similar to Fig. 3(c). One localized defect site is already present near the upper end of the SWNT but the lower part appears nearly defect-free. A localized defect site was photoinduced in this part using the intense and highly confined optical fields underneath the tip, similar to defect generation observed in confocal experiments. ${ }^{13,14}$ From the observed Raman enhancement, we can estimate an excitation enhancement factor of about 20 underneath the tip. ${ }^{10}$ We positioned the tip on the SWNT at position B using a laser power of $500 \mu \mathrm{W}$. Simultaneously, tip-enhanced spectra were acquired every $2 \mathrm{~s}$ to follow the evolution of D-band intensity at this position. The process was stopped after about $100 \mathrm{~s}$ to prevent spatial broadening of the defective spot due to tipdrift. Nevertheless, two closely spaced localized defective spots were created as can be seen in the spectroscopic image recorded afterwards, shown in Figs. 3(b) and 3(c). The D-band intensity $I_{D}$ has strongly increased around position $\mathrm{B}$, whereas the G-Band is still rather uniform along the SWNT, excluding major structural damage. Also the topography did not change considerably (data not shown). Figure 3(e) presents the spectra from before and after the tipinduced defect generation. Both the $I_{D} / I_{G}$-ratio and $I_{D}$ increase strongly and rather continuously with time, as shown in the inset. This means that many defects can be generated within the short nanotube segment that is probed and modified by the tip fields, without an apparent saturation of $I_{D}$. Saturation is expected when the average defect distance approaches $l_{D}$ so that every excited electron or hole can scatter with a defect. Since the probed length is only $15 \mathrm{~nm}, l_{D}$ must be significantly smaller than $5 \mathrm{~nm}$, since otherwise a steplike increase in $I_{D} / I_{G}$ and a fast saturation are expected. The spatial distribution of $I_{D} / I_{G}$ along the SWNT after defect generation is shown in Fig. 3(d). Apparently, the D-band
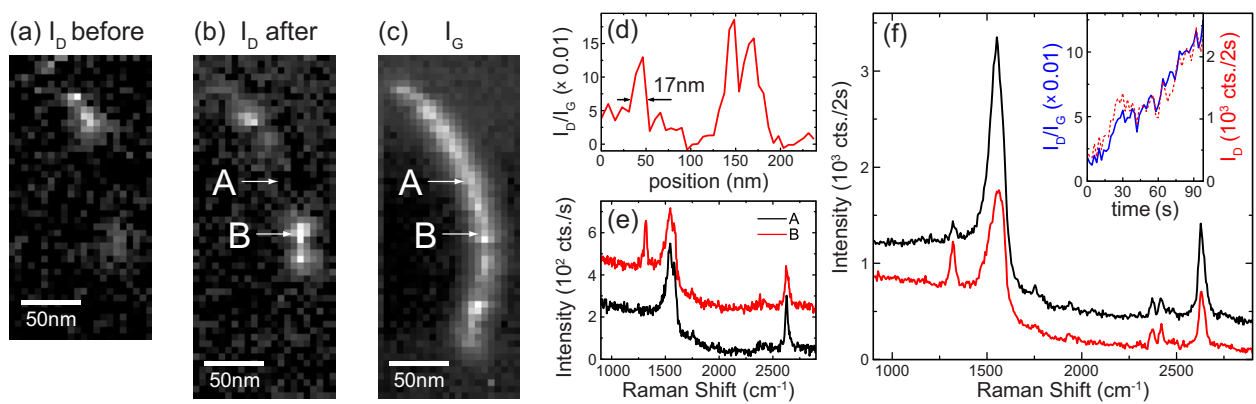

FIG. 3. (Color online) [(a)-(c)] Spectroscopic images of a single SWNT before and after generation of an additional defect site (pixel size is $5.7 \mathrm{~nm})$. (d) Distribution of the $I_{D} / I_{G}$-ratio along the SWNT, showing strong local variations on a length scale of $17 \mathrm{~nm}$. (e) Spectra from positions A and B, representing defect-free and highly defective positions within only $50 \mathrm{~nm}$. (f) Spectrum at position B before (upper) and after (lower curve) the near-field-induced generation of a localized defective region. The inset shows the temporal evolution of $I_{D} / I_{G}$ (solid) and $I_{D}$ (dashed) when the tip was parked on position B. 
scattering arises mainly from three defect sites and is localized within $17 \mathrm{~nm}$ around those. Given the optical resolution of $15 \mathrm{~nm}$, we estimate $l_{D} \leq 2 \mathrm{~nm}$, clearly well below $5 \mathrm{~nm}$. In principle, some defects could also be created during the imaging process but the exposure time ( $1 \mathrm{~s}$ per image pixel) is comparably short.

Regarding the connection between defect density and $I_{D} / I_{G}$-ratio, we can use the model in Ref. 6 developed for graphene and adopt it to one-dimensional SWNTs. For average defect distances $L_{d}>10 \mathrm{~nm}, I_{D} / I_{G}$ is inversely proportional to $L_{d}$, analogous to the $1 / L_{d}^{2}$ dependence in twodimensional graphene. The scaling factor $C$ for the metallic SWNTs studied here can be estimated based on the spectra in Fig. 3(f) and the increase in $I_{D} / I_{G}$ during defect generation. At the beginning, some weak D-band is already detectable, corresponding to at least one defect. The observed eightfold increase after $100 \mathrm{~s}$ requires a minimum of $8 \mathrm{de}-$ fects within the probed length of about $15 \mathrm{~nm}$ corresponding to $L_{d} \approx 2 \mathrm{~nm}$ at $I_{D} / I_{G}=0.12$. This would result in $C$ $=0.24 \mathrm{~nm}$, but the value has to be corrected to about $0.5 \mathrm{~nm}$ using the model, since for $L_{d}<10 \mathrm{~nm}$ the ratio $I_{D} / I_{G}$ already deviates from the inverse linear scaling. We thus derive the relation $L_{d} \approx 0.5 \mathrm{~nm} \times\left(I_{D} / I_{G}\right)^{-1}$, from which the defect density can be determined by measuring $I_{D} / I_{G}$. Determining $L_{d}$ in SWNTs using the relation established for nanographite ${ }^{15}$ is probably inaccurate, since the respective scaling factor is about 75 times larger.

The type of defects that lead to the observed D-band cannot be specified in our experiment. We do neither see changes in the shape of the G-band characteristic for charged defects ${ }^{16}$ nor do we observe significant shifts in the G-band. Structural defects from the growth process and photoinduced local oxidation of the SWNT could be a reasonable explanation. In general, the nanotube material studied here exhibits only few defects per micrometer. Importantly, the ends of the SWNTs do not show apparent D-band scattering in contrast to the armchair edge of graphene. In the case of SWNTs the D-band intensity connected to the edge could be very weak since the selection rules requiring a certain edge type and especially light polarization parallel to the edge will be rarely fulfilled. ${ }^{5}$ Furthermore, the end probably acts only as a single defect, which will be very hard to detect.
In conclusion, we used tip-enhanced Raman spectroscopy to investigate the D-band scattering near defects in metallic SWNTs with high spatial resolution. We found that the length scale of the D-band scattering process $l_{D}$ is about $2 \mathrm{~nm}$, similar as for graphene. ${ }^{5,6}$ The knowledge of $l_{D}$ allows to determine the average defect distance $L_{d}$ from the $I_{D} / I_{G}$-ratio via the relation $L_{d}=0.5 \mathrm{~nm} \cdot\left(I_{D} / I_{G}\right)^{-1}$ estimated for the investigated metallic SWNTs.

We thank Nanointegris for generous support with SWNT material. The work was funded by the Deutsche Forschungsgemeinschaft (Grant No. DFG-HA4405/3-1) and the Nanosystems Initiative Munich (NIM).

${ }^{1}$ M. S. Dresselhaus, A. Jorio, M. Hofmann, G. Dresselhaus, and R. Saito, Nano Lett. 10, 751 (2010).

${ }^{2}$ J. Maultzsch, S. Reich, and C. Thomsen, Phys. Rev. B 64, 121407(R) (2001).

${ }^{3}$ A. C. Ferrari, Solid State Commun. 143, 47 (2007).

${ }^{4}$ F. Tuinstra and J. L. Koenig, J. Chem. Phys. 53, 1126 (1970).

${ }^{5}$ C. Casiraghi, A. Hartschuh, H. Qian, S. Piscanec, C. Georgi, A. Fasoli, K. S. Novoselov, D. M. Basko, and A. Ferrari, Nano Lett. 9, 1433 (2009).

${ }^{6}$ M. M. Lucchese, F. Stavale, E. H. Martins Ferreira, C. Vilani, M. V. O. Moutinho, R. B. Capaz, C. A. Achete, and A. Jorio, Carbon 48, 1592 (2010).

${ }^{7}$ L. G. Cançado, R. Beams, and L. Novotny, arXiv:0802.3709v1 (unpublished).

${ }^{8}$ A. K. Gupta, T. J. Russin, H. R. Gutiérrez, and P. C. Eklund, ACS Nano 3, 45 (2009).

${ }^{9}$ A. Hartschuh, E. J. Sánchez, X. S. Xie, and L. Novotny, Phys. Rev. Lett. 90, 095503 (2003)

${ }^{10}$ A. Hartschuh, Angew. Chem., Int. Ed. 47, 8178 (2008).

${ }^{11}$ L. G. Cançado, A. Jorio, A. Ismach, E. Joselevich, A. Hartschuh, and L. Novotny, Phys. Rev. Lett. 103, 186101 (2009).

${ }^{12}$ M. S. Arnold, A. A. Green, J. F. Hulvat, S. I. Stupp, and M. C. Hersam, Nat. Nanotechnol. 1, 60 (2006).

${ }^{13}$ C. Georgi, N. Hartmann, T. Gokus, A. A. Green, M. C. Hersam, and A. Hartschuh, ChemPhysChem 9, 1460 (2008).

${ }^{14}$ H. Harutyunyan, T. Gokus, A. A. Green, M. C. Hersam, M. Allegrini, and A. Hartschuh, Nano Lett. 9, 2010 (2009).

${ }^{15}$ L. G. Cançado, K. Takai, T. Enoki, M. Endo, Y. A. Kim, H. Mizusaki, A. Jorio, L. N. Coelho, R. Magalhães-Paniago, and M. A. Pimenta, Appl. Phys. Lett. 88, 163106 (2006).

${ }^{16}$ I. O. Maciel, N. Anderson, M. A. Pimenta, A. Hartschuh, H. Qian, M. Terrones, H. Terrones, J. Campos-Delgado, A. M. Rao, L. Novotny, and A. Jorio, Nature Mater. 7, 878 (2008). 